\title{
Two Loci of Expression for Long-Term Depression at Hippocampal Mossy Fiber-Interneuron Synapses
}

\author{
Saobo Lei and Chris J. McBain \\ Laboratory of Cellular and Synaptic Neurophysiology, National Institute of Child Health and Human Development, National Institutes of Health, Bethesda, \\ Maryland 20892-4495
}

Two distinct forms of long-term depression (LTD) exist at mossy fiber synapses between dentate gyrus granule cells and hippocampal CA3 stratum lucidum interneurons. Although induction of each form of LTD requires an elevation of postsynaptic intracellular $\mathrm{Ca}^{2+}{ }^{2}$, at $\mathrm{Ca}^{2+}$-impermeable AMPA receptor (CI-AMPAR) synapses, induction is NMDA receptor (NMDAR) dependent, whereas LTD at Ca ${ }^{2+}$ permeable AMPA receptor (CP-AMPAR) synapses is NMDAR independent. However, the expression locus of either form of LTD is not known. Using a number of criteria, including the coefficient of variation, paired-pulse ratio, AMPA-NMDA receptor activity, and the low-affinity AMPAR antagonist $\gamma$-D-glutamyl-glycine, we demonstrate that LTD expression at CP-AMPAR synapses is presynaptic and results from reduced transmitter release, whereas LTD expression at CI-AMPAR synapses is postsynaptic. The $N$-ethylmaleimidesensitive fusion protein-AP2-clathrin adaptor protein 2 inhibitory peptide pep2m occluded LTD expression at CI-AMPAR synapses but not at CP-AMPAR synapses, confirming that CI-AMPAR LTD involves postsynaptic AMPAR trafficking. Thus, mossy fiber innervation of CA3 stratum lucidum interneurons occurs via two parallel systems targeted to either $\mathrm{Ca}^{2+}$-permeable or $\mathrm{Ca}^{2+}$-impermeable AMPA receptors, each with a distinct expression locus for long-term synaptic plasticity.

Key words: AMPA; depression; hippocampus; interneuron; synapse; glutamate

\section{Introduction}

The mossy fiber axons of dentate gyrus granule cells innervate CA3 pyramidal neurons via large, complex boutons with multiple release sites, whereas inhibitory interneurons of the CA3 stratum lucidum receive innervation via small en passant or filopodial extensions that often comprise a single release site (Acsady et al., 1998). AMPA receptors (AMPARs) at mossy fiber-interneuron synapses comprise a continuum that ranges from glutamate receptor 2 (GluR2)-lacking, $\mathrm{Ca}^{2+}$-permeable (CP)-AMPAR synapses to GluR2-containing, $\mathrm{Ca}^{2+}$-impermeable (CI)-AMPAR synapses (Toth et al., 2000; Bischofberger and Jonas, 2002; Lawrence and McBain, 2003). NMDA receptor (NMDAR) expression also differs between the two extremes of this continuum, which in part provide distinct mechanisms for mossy fiber excitation of postsynaptic inhibitory interneurons (Lei and McBain, 2002).

High-frequency mossy fiber stimulation induces long-term potentiation at CA3 pyramidal neuron synapses (Harris and Cotman, 1986; Zalutsky and Nicoll, 1990; Nicoll and Malenka, 1995; Yeckel et al., 1999), whereas, the same stimulation paradigm in-

Received 0ct. 14, 2003; revised Dec. 17, 2003; accepted Jan. 6, 2004.

C.J.M. was supported by the National Institute of Child Health and Human Development Intramural Research Program and the Human Frontiers Science Program (C.J.M.). We thank Drs. John Isaacs and Jeremy Henley for discussions concerning the NSF inhibitory peptide and Drs. Josh Lawrence and Jeff Diamond for their constructive criticism of this manuscript.

Correspondence should be addressed to Chris J. McBain, Laboratory of Cellular and Synaptic Neurophysiology, National Institutes of Health, Room 5A72, Building 49, Convent Drive, Bethesda, MD 20892. E-mail: mcbainc@mail.nih.gov.

DOI:10.1523/JNEUROSCI.4645-03.2004

Copyright $\odot 2004$ Society for Neuroscience $\quad$ 0270-6474/04/242112-10\$15.00/0 duces two forms of long-term depression (LTD) at mossy fiberCA3 interneuron synapses. Each form of LTD is associated with a distinct AMPAR synapse type (Toth et al., 2000; Lei and McBain, 2002). Induction of LTD at CI-AMPAR synapses requires activation of NMDARs and an elevation of postsynaptic $\mathrm{Ca}^{2+}$. In contrast, although LTD at CP-AMPAR synapses also requires a postsynaptic $\mathrm{Ca}^{2+}$ elevation, induction is NMDAR independent (Laezza et al., 1999). This differential dependence of LTD induction on NMDAR activation likely arises as a consequence of the dissimilar NMDAR:AMPAR ratios present at either synapse and the resulting sources of $\mathrm{Ca}^{2+}$ (Bischofberger and Jonas, 2002; Lei and McBain, 2002).

In the hippocampus, LTD expression at principal neuron synapses is typically associated with a reduction in presynaptic transmitter release, a reduction in postsynaptic AMPAR density, or sensitivity to a fixed concentration of glutamate (Sheng and Kim, 2002; Song and Huganir, 2002). At hippocampal interneuron synapses, the expression locus for either form of LTD is unexplored, but evidence suggests that LTD at mossy fiber-dentate gyrus basket cell synapses has a presynaptic expression locus (Alle et al., 2001). In the present study, we investigated the expression locus of both forms of mossy fiber-interneuron LTD using three approaches. First, we used conventional methods, including measuring the coefficient of variation $(\mathrm{CV})$, the paired-pulse ratio (PPR), and NMDAR activity, to assess potential changes in presynaptic transmitter release probability. Then we probed the LTD expression locus further using a low-affinity, competitive AMPAR antagonist, $\gamma$-D-glutamyl-glycine $(\gamma$-DGG), to detect potential changes in transmitter profile in the cleft (Liu et al., 1999; Wadiche and Jahr, 2001; Shen et al., 2002). Finally, inde- 
pendent confirmation of these results came from experiments demonstrating that NMDAR-dependent LTD was occluded by postsynaptic infusion of an $\mathrm{N}$-ethylmaleimide-sensitive fusion protein (NSF)-AP2-clathrin adaptor protein inhibitory peptide, pep2m (KRMKVAKNAQ) (Lüscher et al., 1999; Lüthi et al., 1999; Lee et al., 2002), suggesting a mechanism involving postsynaptic AMPAR trafficking at CI-AMPAR synapses. In contrast, pep2m neither changed the amplitude of control synaptic transmission nor occluded LTD expression at CP-AMPAR synapses, further supporting a presynaptic expression locus.

\section{Materials and Methods}

Hippocampal slice preparation. Transverse hippocampal slices $(300 \mu \mathrm{m})$ were obtained from 16- to 20-d-old Sprague Dawley rats, as described previously (Lei and McBain, 2002, 2003). Briefly, rats were deeply anesthetized with isoflurane and rapidly decapitated. The brain was dissected out in ice-cold saline solution that contained the following (in $\mathrm{mM}$ ): 130 $\mathrm{NaCl}, 24 \mathrm{NaHCO}_{3}, 3.5 \mathrm{KCl}, 1.25 \mathrm{NaH}_{2} \mathrm{PO}_{4}, 1.0 \mathrm{CaCl}_{2}, 5.0 \mathrm{MgCl}_{2}$, and 10 glucose, saturated with $95 \% \mathrm{O}_{2}$ and $5 \% \mathrm{CO}_{2}$, $\mathrm{pH}$ 7.4. All animal procedures conformed to National Institutes of Health animal welfare guidelines. Slices were incubated in the same solution for periods of at least $1 \mathrm{hr}$ at room temperature before their use.

Electrophysiology. Whole-cell patch-clamp recordings were made at room temperature $\left(\sim 22-24^{\circ} \mathrm{C}\right)$ from visually identified interneurons located within the stratum lucidum of CA3 using an Axopatch 200A amplifier (Axon Instruments, Foster City, CA) in voltage-clamp mode (Lei and McBain, 2002). Recording electrodes were filled with the following solution (in mM): 100 Cs-gluconate, 0.6 EGTA, $5 \mathrm{MgCl}_{2}, 8 \mathrm{NaCl}, 2$ $\mathrm{ATP}_{2} \mathrm{Na}, 0.3 \mathrm{GTPNa}, 40$ HEPES, 0.4 spermine, and 1 QX-314, $(\mathrm{N}-(2,6-$ dimethylphenylcarbomoylmethyltriethylammonium bromide), $\mathrm{pH}$ 7.27.3. Biocytin $(0.2 \%)$ was routinely added to the recording electrode solution to allow post hoc morphological processing of recorded cells. Unless otherwise stated, the extracellular solution comprised the following composition (in mM): $130 \mathrm{NaCl}, 24 \mathrm{NaHCO}_{3}, 3.5 \mathrm{KCl}, 1.25 \mathrm{NaHPO}_{4}$, $1.5 \mathrm{MgCl}_{2}, 2.5 \mathrm{CaCl}_{2}, 10$ glucose, and 0.1 bicuculline methobromide, saturated with $95 \% \mathrm{O}_{2}$ and $5 \% \mathrm{CO}_{2}, \mathrm{pH} 7.4$. At the end of all recordings, the group II metabotropic GluR (mGluR) agonist $\left(2 S, 2^{\prime} R, 3^{\prime} R\right)-2-\left(2^{\prime}, 3^{\prime}-\right.$ dicarbocyclopropyl) glycine (DCG-IV) $(1 \mu \mathrm{M})$ was added to the perfusate to confirm that synaptic events were mossy fiber in origin (Kamiya et al., 1996; Toth and McBain, 1998; Lawrence et al., 2004). Series resistance was rigorously monitored by the delivery of $5 \mathrm{mV}$ voltage steps after each evoked EPSC. Experiments were discontinued if the series resistance changed by $>15 \%$. LTD of evoked synaptic transmission was induced by a high-frequency stimulation (HFS) paradigm ( $100 \mathrm{~Hz}$ for $1 \mathrm{sec}$, repeated three times at an interval of $10 \mathrm{sec}$ ).

Synaptic responses were evoked by low-intensity stimulation (duration, $80 \mu \mathrm{sec}$; intensity, $40-80 \mu \mathrm{A}$ ) of dentate gyrus granule cells or by placement directly in the stratum lucidum of the CA3 hippocampus via a constant-current isolation unit (A360; World Precision Instruments, Sarasota, FL) connected to a patch electrode filled with oxygenated extracellular solution. For each experiment, the stimulus intensity was set to the lowest value that reliably evoked a multifiber EPSC waveform with minimal failures; no attempt was made to stimulate single fiber responses. Synaptic responses were included in the analysis if the rise times and decay time constants were monotonic and possessed no apparent polysynaptic waveforms. In most experiments, a solution containing (in $\mu \mathrm{M}) 100 \mathrm{dl}$-APV, 100 bicuculline, and $10 \mathrm{DNQX}$ was added at the end of the experiment to record the isolated stimulus artifact, which was then averaged and subtracted from synaptic records to obtain stimulus artifact-free records of EPSCs for accurate amplitude measurement. In those experiments in which this was not possible, the stimulus artifact was blanked from the trace for clarity. Rise times were measured as $20-80 \%$ duration. Current traces shown in the figures are the averages of $10-15$ EPSCs recorded at the corresponding time points indicated in the figures.

In experiments designed to monitor changes in NMDAR activity before and after LTD expression (see Fig. 2A1,B1), we initially recorded the composite-evoked EPSC mediated by both NMDARs and AMPARs at a holding potential of $+40 \mathrm{mV}$ for $1 \mathrm{~min}$ (20 EPSCs) and then switched the holding potential to $-60 \mathrm{mV}$ to record the basal AMPA EPSC. LTD was then induced by HFS, and after a period to allow stabilization of plasticity, the composite EPSCs mediated by both NMDARs and AMPARs were again recorded at $+40 \mathrm{mV}$. The $\mathrm{Ca}^{2+}$-permeable nature of AMPARs was then identified by bath application of philanthotoxin (Toth et al., 2000; Lei and McBain, 2002). A time point $50 \mathrm{msec}$ after the end of the stimulation artifact was chosen for measurement of the NMDAR component, because at this time point, NMDARs were still open, but AMPARs were almost completely deactivated.

Data analysis. The rectification index (RI) of the evoked EPSC was generated from averaged (20-30 traces) AMPA EPSC amplitudes at a series of holding potentials between -60 and $+40 \mathrm{mV}$ (Lei and McBain, 2002). EPSC amplitudes recorded at negative holding potentials (from -60 to $-20 \mathrm{mV}$ ) were fit by a linear regression. The RI of the $I-V$ relationship was then defined as the ratio of the actual current amplitude at $+40 \mathrm{mV}$ to the predicted linear value at $+40 \mathrm{mV}$ (Liu and Cull-Candy, 2000). The PPR was calculated as the mean P2/mean P1 (Kim and Alger, 2001), where P1 was the amplitude of the first evoked current and P2 was the amplitude of the second synaptic current, measured after subtraction of the remaining P1 "tail" current. The CV of synaptic currents was calculated as the SD of current amplitude divided by mean $(x)$ of the current amplitude $(\mathrm{CV}=\mathrm{SD} / x)$.

Data are presented as means \pm SEM and, unless stated otherwise, were analyzed using a paired Student's $t$ test; $p$ values are reported throughout Results.

Chemicals. $\gamma$-DGG was purchased from Tocris (Ellisville, MO). Pep2m and pep- $\Delta$ A849-Q853 (Lee et al., 2002) were synthesized by Sigma (St. Louis, MO) (purity, $>95 \%$ ). All other chemicals were products of Sigma.

\section{Results}

LTD induction at mossy fiber-CP-AMPAR and -CI-AMPAR interneuron synapses requires postsynaptic calcium elevation (Lei and McBain, 2002). However, the locus and mechanism(s) of LTD expression at either synapse type are completely unexplored. In the first series of experiments, we used conventional methods of analysis to explore the presynaptic or postsynaptic locus of each type of mossy fiber-interneuron LTD.

Mossy fiber EPSCs were evoked $\left(V_{\text {hold }}=-60 \mathrm{mV}, 0.33 \mathrm{~Hz}\right.$ ) by placement of a stimulating electrode in the dentate gyrus granule cell layer or in the stratum lucidum (Toth and McBain, 1998; Lei and McBain, 2002). The RI of AMPARs was used to identify CI-AMPAR and CP-AMPAR synapses using criteria established previously (Toth and McBain, 1998; Lei and McBain, 2002). We defined AMPA EPSCs possessing an RI of $>0.7$ as CI-AMPAR synapses and those with an RI of $<0.3$ as CP-AMPAR synapses. At the end of all recordings, the group II mGluR agonist DCG-IV $(1 \mu \mathrm{M})$ was added to the perfusate to reduce evoked synaptic events confirming their mossy fiber origin (Kamiya et al., 1996; Lei and McBain, 2002; Lawrence et al., 2004). Although biocytin filling confirmed that all cells recovered were stratum lucidum interneurons, attempts to correlate the presence of CI-AMPAR LTD or CP-AMPAR LTD with cell morphology proved inconclusive when based solely on the somatodendritic-axonal arbors. Consistent with our previous observations (Toth and McBain, 1998), CP-AMPARs and CI-AMPARs were found on almost all morphologically defined subgroups of the stratum lucidum interneuron.

\section{LTD expression does not change the rectification index of AMPA receptors}

In cerebellar interneurons, the subunit composition of postsynaptic AMPARs is activity dependent, such that repetitive synaptic activation of $\mathrm{Ca}^{2+}$-permeable AMPARs rapidly reduces $\mathrm{Ca}^{2+}$ 
A

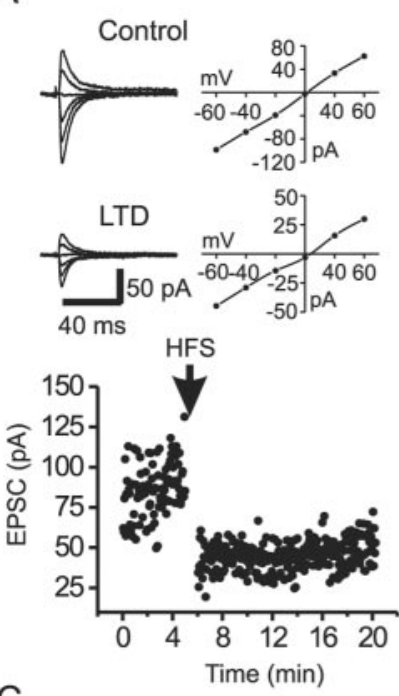

C
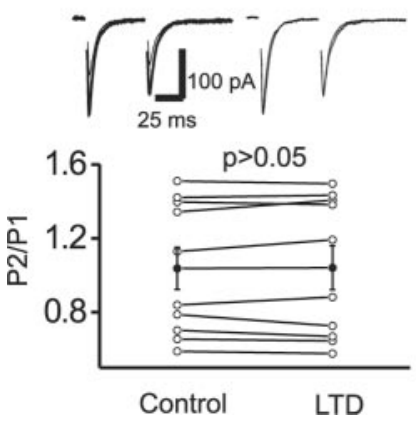

B

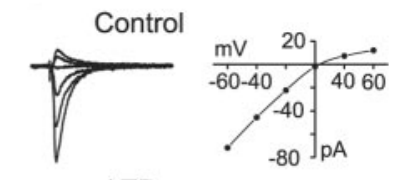

LTD
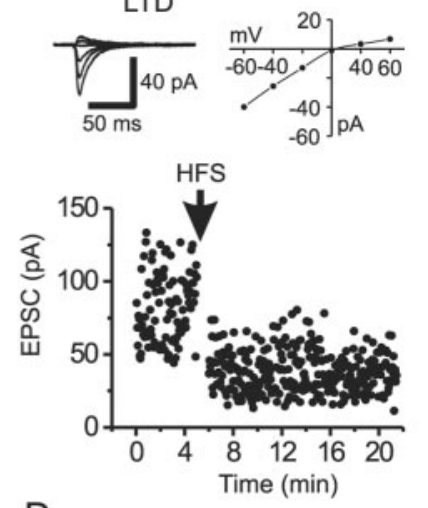

D
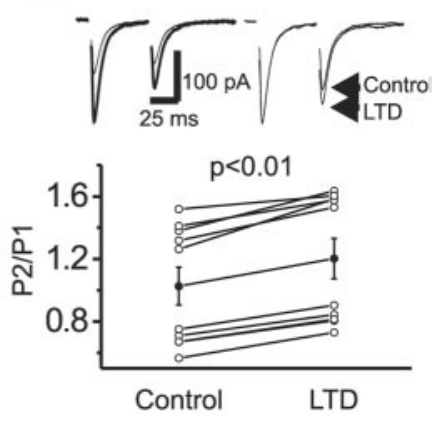

Figure 1. LTD expression does not change the RI of either CI-AMPAR or CP-AMPAR synapses but increases PPR at (P-AMPAR synapses. $A, B$, The rectification index is unaltered after LTD expression at both (I-AMPAR ( $A$ ) and CP-AMPAR ( $B$ ) synapses. Top, Control AMPAR-mediated EPSCs evoked at different holding potentials (left) and their corresponding I- $V$ relationship (right). Middle, AMPAR EPSCs (left) and corresponding $/-V$ curve (right) after LTD expression. Bottom, Time course of LTD at each synapse. C, D, LTD expression does not change the PPR at CI-AMPAR synapses ( $C$ ) but increases the PPR at (P-AMPAR synapses ( $D$ ). Top left, Paired EPSC evoked by two stimuli at an interval of $50 \mathrm{msec}$ before (thick) and after (thin) LTD expression. Top right, EPSCs recorded before and after LTD expression normalized to the first EPSC peak. Note the change in PPR at the (P-AMPAR synapse. Bottom, Pooled data for PPRs from different synapses (open circles) and pooled mean data (filled circles). Note that LTD expression significantly increases PPR only at CP-AMPAR synapses.

permeability and changes EPSC amplitude by the selective incorporation of GluR2-containing AMPARs (Liu and Cull-Candy, 2000, 2002). For the experiments that follow, it was important to determine whether a similar mechanism exists at mossy fiberinterneuron synapses (i.e., does LTD expression alter the relative $\mathrm{Ca}^{2+}$ permeability of evoked synaptic events?). HFS of mossy fibers $(100 \mathrm{~Hz}$ for $1 \mathrm{sec}$, repeated three times at an interval of 10 sec) induced an LTD (Fig. 1A,B) similar to that described previously (Maccaferri et al., 1998; Toth et al., 2000; Lei and McBain, 2002). After LTD expression, the RI was not significantly altered at either CI-AMPAR (control, $0.94 \pm 0.05$; LTD, $0.97 \pm 0.05 ; p=$ $0.67 ; n=9$ ) (Fig. $1 A$ ) or CP-AMPAR (control, $0.17 \pm 0.03$; LTD, $0.21 \pm 0.02 ; p=0.40 ; n=6$ ) (Fig. $1 B$ ) synapses, suggesting that the molecular composition and $\mathrm{Ca}^{2+}$ permeability of AMPARs are unchanged after LTD expression.

LTD expression locus differentiated by $\mathrm{CV}$ and PPR analysis To determine whether LTD expression at CI-AMPAR and CPAMPAR synapses shares a similar locus, we compared the $\mathrm{CV}$ and

the PPR of AMPA EPSCs before and after LTD expression. At CI-AMPAR synapses, the $\mathrm{CV}$ was unaltered after LTD expression (control, $0.37 \pm 0.04 ;$ LTD, $0.39 \pm 0.05 ; n=9 ; p=0.29$ ) (data not shown), whereas LTD expression at CP-AMPAR synapses was associated with an increased CV (control, $0.34 \pm 0.06$; LTD, $0.50 \pm 0.08 ; n=6 ; p=0.01$ ), suggesting that LTD at CPAMPARs but not CI-AMPARs may be related to changes in presynaptic transmitter release.

We next examined the PPR (mean P2/mean P1) (Kim and Alger, 2001) before and after LTD expression. Consistent with previous results (Toth et al., 2000), either paired-pulse depression (PPD) or paired-pulse facilitation (PPF) was detected at both CI-AMPAR and CP-AMPAR synapses (Fig. $1 C, D$ ). In all CI-AMPAR synapses tested, the mean PPR was not altered after LTD expression (control, $1.04 \pm 0.11$; LTD, $1.04 \pm 0.12 ; n=10$; $p=0.79$ ) (Fig. 1C). However, the mean PPR was significantly increased after LTD expression at CP-AMPAR synapses (control, $1.03 \pm 0.12$; LTD, $1.20 \pm 0.13 ; n=10 ; p=0.0001$ ) (Fig. $1 D$ ).

\section{LTD expression locus evaluated by NMDAR activity}

If LTD expression at CP-AMPAR synapses arises from a reduction in presynaptic glutamate release, then a reduction in the NMDAR-mediated EPSCs concomitant with changes in both CV and PPR would also be expected. Representative experiments from CI-AMPAR and CP-AMPAR synapses are shown in Figure $2, A 1$ and $B 1$, respectively. Cells were held at $+40 \mathrm{mV}$ to measure the initial NMDAR-mediated component (measured at $50 \mathrm{msec}$ after the stimulus artifact). Cells were then voltage clamped at $-60 \mathrm{mV}$, and LTD was induced by the HFS paradigm. After LTD expression (15-20 $\mathrm{min}$ ), cells were again voltage clamped at +40 $\mathrm{mV}$ to monitor changes in NMDAR EPSC. At both synapse types, the NMDAR component was significantly reduced after LTD expression (Fig. $2 A 1, A 2, B 1, B 2$ ). Similar to AMPA EPSCs, the CV of NMDA EPSCs at CI-AMPAR synapses was not significantly altered after LTD expression (Fig. $2 \mathrm{A3}$ ), whereas the CV of NMDA EPSCs at CP-AMPAR synapses was significantly increased (Fig. 2B3). Together, these data suggest that LTD expression at CPAMPAR synapses is consistent with a presynaptic locus, whereas LTD expression at CI-AMPAR synapses may be postsynaptic.

\section{Changes in synaptic glutamate concentration probed with the low-affinity AMPAR antagonist $\gamma$-DGG}

Next, we explored the nature of transmitter release at mossy fiber-interneuron synapses. Taking advantage of the low-affinity, competitive AMPAR antagonist $\gamma$-DGG, we probed for variations in transmitter glutamate concentration during paired-pulse paradigms and after LTD expression at both synapse types (Liu et al., 1999; Wadiche and Jahr, 2001; Shen et al., 2002). These experiments are based on the observation that a certain fraction of synaptic receptors continually exposed to $\gamma$-DGG and then subjected to the rapid synaptic glutamate transient will replace bound $\gamma$-DGG for glutamate because of the low affinity of $\gamma$-DGG. Consequently, the degree of $\gamma$-DGG inhibition can be used to assess changes in the glutamate transient concentration in the synaptic cleft during paired-pulse protocols (Wadiche and Jahr, 2001) and after induction of LTD (Shen et al., 2002).

First, to confirm that changes in $\gamma$-DGG inhibition reliably reflect alterations in cleft glutamate concentration, we changed transmitter release probability by altering the extracellular $\mathrm{Ca}^{2+}$ concentration $\left(\left[\mathrm{Ca}^{2+}\right]_{\mathrm{o}}\right)$ and examined the corresponding changes in $\gamma$-DGG (1 mM) inhibition of evoked EPSCs. When $\left[\mathrm{Ca}^{2+}\right]_{\mathrm{o}}$ was elevated from 2.5 to $4 \mathrm{~mm}$, the magnitude of $\gamma$-DGG inhibition was significantly reduced at both CI-AMPAR (39.9 \pm 
A1

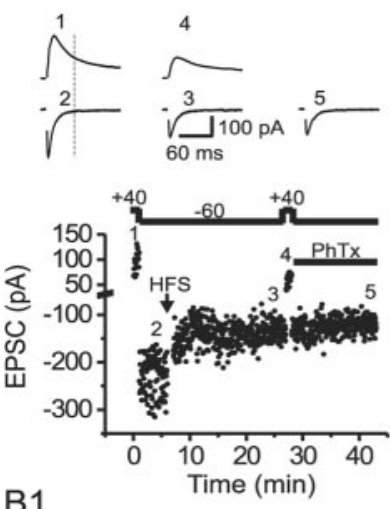

B1

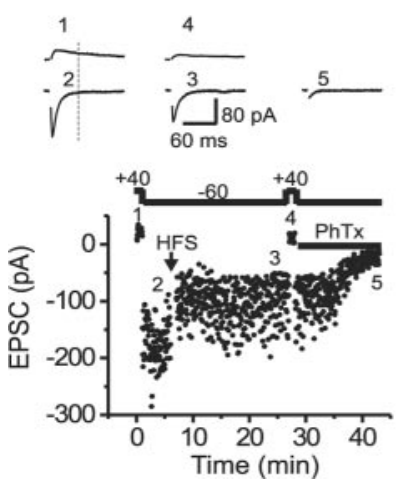

A2
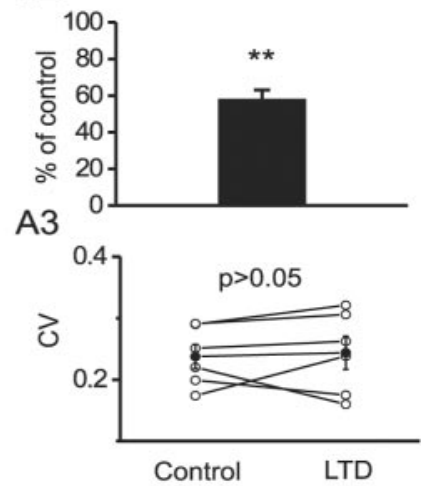

B2

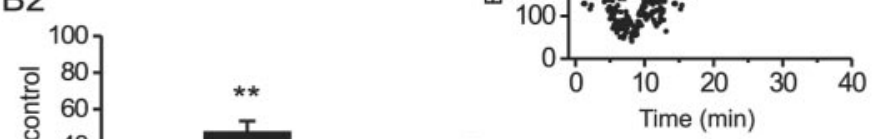

B3

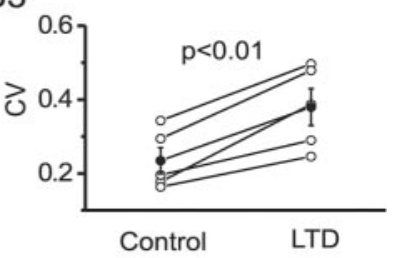

B

A
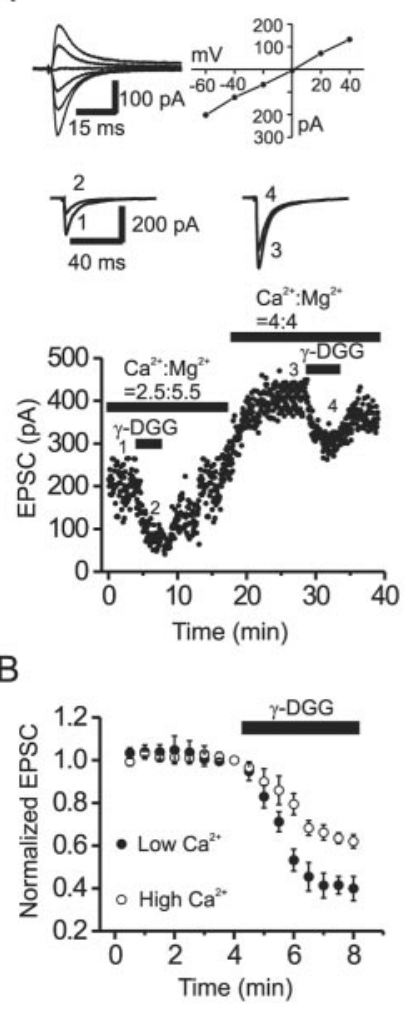

C
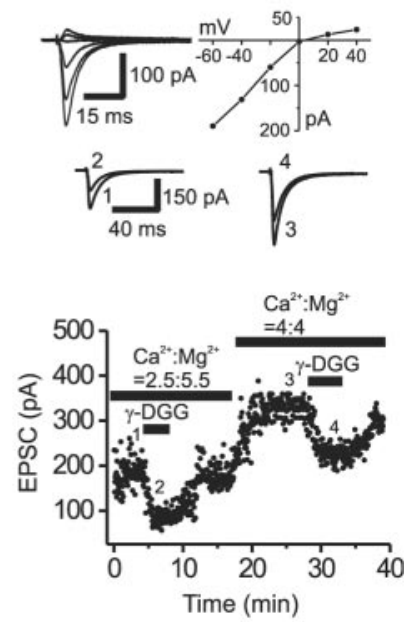

D

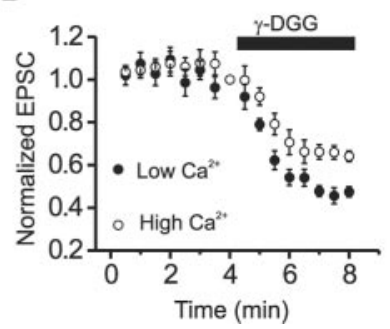

Figure 2. LTD expression is correlated with a reduction in NMDAR activity at both CI-AMPAR and CP-AMPAR synapses, but an increase in CV was observed only at CP-AMPAR synapses. A1, Time course of EPSCs recorded at $+40 \mathrm{mV}$ ( 1 and 4$)$ or $-60 \mathrm{mV}(2,3$, and 5) from a (I-AMPAR synapse. Traces in the top panels are EPSCs recorded at the time points indicated in the dot plot (bottom). In this series of experiments, philanthotoxin ( $\mathrm{PhT} ; 5 \mu \mathrm{M}$ ) was added at the end of the experiment to confirm the $\mathrm{Ca}^{2+}$-permeable nature of the synapse being studied. $A 2$, The mean NMDAR-mediated EPSC (measured at $50 \mathrm{msec}$ after the stimulus artifact indicated by the vertical dotted lines in both $A 1$ and $B 1$ ) is significantly reduced after LTD expression at (I-AMPAR synapses. $A 3$, The mean NMDAR-EPSC CV is unaltered after LTD expression at CI-AMPAR synapses. B1-B3, Corresponding data from CP-AMPAR synapses arranged as described above for CI-AMPAR synapses. B3, Note that in addition to a reduction in the NMDAR-EPSC amplitude, the NMDAR-EPSC CV was significantly increased after LTD expression. ${ }^{* *} p<0.01$.

5.7 vs $62.0 \pm 3.1 \%$ of controls; $n=5 ; p=0.003$ ) (Fig. $3 A, B$ ) and CP-AMPAR $(47.5 \pm 2.4$ vs $64.1 \pm 2.5 \%$ of controls; $n=5 ; p=$ 0.002 ) (Fig. $3 C, D$ ) synapses. However, a similar increase in $\left[\mathrm{Ca}^{2+}\right]_{\mathrm{o}}$ failed to alter the magnitude of EPSC inhibition by the high-affinity AMPAR antagonist DNQX $(0.2 \mu \mathrm{M})$ at either CI$\operatorname{AMPAR}(48.9 \pm 4.2$ vs $45.9 \pm 3.8 \% ; n=4 ; p=0.67)$ or $\mathrm{CP}$ $\operatorname{AMPAR}(51.2 \pm 5.3$ vs $47.4 \pm 3.3 \% ; n=3 ; p=0.53)$ synapses (data not shown). Conversely, reducing $\left[\mathrm{Ca}^{2+}\right]_{\mathrm{o}}$ from 2.5 to 0.5 mM significantly increased $\gamma$-DGG inhibition at both CI-AMPAR $(47.4 \pm 2.8$ vs $22.6 \pm 2.7 \%$ of controls; $n=5 ; p=0.004)$ and CP-AMPAR $(50.5 \pm 4.4 \%$ vs $21.6 \pm 3.8 \%$ of controls; $n=4 ; p=$ 0.003 ) synapses (data not shown). Again, this manipulation failed to change the percentage of inhibition by the high-affinity antagonist DNQX at either CI-AMPAR $(49.3 \pm 4.1$ vs $50.2 \pm$ $3.9 \% ; n=3 ; p=0.89)$ or CP-AMPAR (53.1 \pm 4.4 vs $48.8 \pm 3.7 \%$; $n=3 ; p=0.76)$ synapses. The above results are consistent with previous reports (Liu et al., 1999; Wadiche and Jahr, 2001; Shen et al., 2002) and suggest that the magnitude of inhibition by the low-affinity antagonist $\gamma$-DGG (but not the high-affinity antagonist) is sensitive to variation in synaptic glutamate concentration at both CP-AMPAR and CI-AMPAR synapses.

We next tested whether the magnitude of $\gamma$-DGG block de-

Figure 3. Increasing transmitter release probability reduces the magnitude of $\gamma$-DGG inhibition at both Cl-AMPAR and CP-AMPAR synapses. A, Dot plot shows the time course of EPSC amplitude from a Cl-AMPAR synapse recorded in the presence of low $(2.5 \mathrm{~mm})$ and high $(4 \mathrm{~mm})$ extracellular $\mathrm{Ca}^{2+}$. Top, AMPA EPSCs recorded at different holding potentials (left) and the corresponding I-V curve (right) from a Cl-AMPAR synapse. Middle, AMPA EPSCs recorded at the time points indicated in the dot plot (bottom). B, Normalized time course of $\gamma$-DGG (1 mM) inhibition in low and high $\left[\mathrm{Ca}^{2+}\right]_{0}$ from five representative Cl-AMPAR synapses. Note that the magnitude of $\gamma$-DGG block is significantly less in elevated extracellular $\mathrm{Ca}^{2+} \cdot C, D$, The corresponding data from CP-AMPAR synapses arranged as described above for Cl-AMPAR synapses.

tected changes in synaptic cleft glutamate concentration during paired-pulse stimulation. Because interneuron synapses possess either facilitation or depression in response to paired-pulse stimulation (Fig. 1C,D) (Toth et al., 2000), we treated the data separately. For CI-AMPAR synapses displaying PPD, application of $\gamma$-DGG $(1 \mathrm{~mm})$ inhibited EPSC $_{1}$ by $47.2 \pm 1.8 \%$ and EPSC $_{2}$ by $57.8 \pm 0.7 \%(n=5 ; p=0.001)$ (Fig. $\left.4 A_{1}\right)$. The larger degree of $\gamma$-DGG block suggests that a lower glutamate transient is evoked by the second stimulus. Consistent with previous results (Liu et al., 1999; Wadiche and Jahr, 2001), $\gamma$-DGG increased the EPSC $_{1}$ and $\mathrm{EPSC}_{2}$ rise times. In addition, $\gamma$-DGG decreased the weighted decay kinetics of $\mathrm{EPSC}_{1}$ (Table 1, Fig. $4 A_{1}$, middle) without significantly changing the decay kinetics of $\mathrm{EPSC}_{2}$. This faster rate of $\mathrm{EPSC}_{1}$ decay in the presence of $\gamma$-DGG likely arises from the more effective block by $\gamma$-DGG of the synaptic tail current, resulting from lower concentrations of glutamate, compared with those occurring at the EPSC peak (Wadiche and Jahr, 2001). For CI-AMPAR synapses exhibiting PPF, $\gamma$-DGG inhibited EPSC $_{1}$ by $44.5 \pm 1.4 \%$ and EPSC $_{2}$ by $37.6 \pm 1.5 \%(n=5 ; p=$ 0.007) (Fig. $4 A_{2}$ ). Similar to synapses showing PPD, $\gamma$-DGG slowed the rise times of EPSC ${ }_{1}$ and $\mathrm{EPSC}_{2}$ without changing their decay kinetics (Table 1).

For CP-AMPAR synapses showing PPD, $\gamma$-DGG inhibition of $\mathrm{EPSC}_{2}(44.5 \pm 1.3 \%)$ was significantly larger than $\mathrm{EPSC}_{1}(29.7 \pm$ $1.6 \% ; n=5 ; p=0.002$ ) (Fig. $4 B_{1}$ ). Similarly, $\gamma$-DGG increased 
$A_{1}$
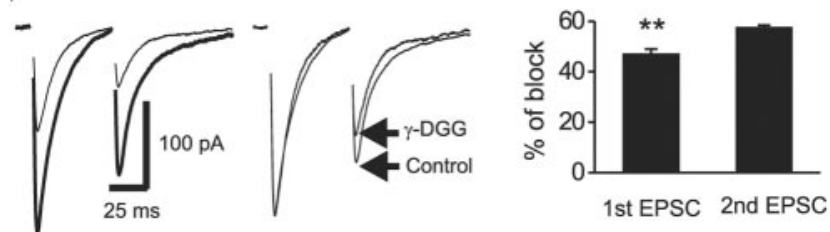

$\mathrm{A}_{2}$

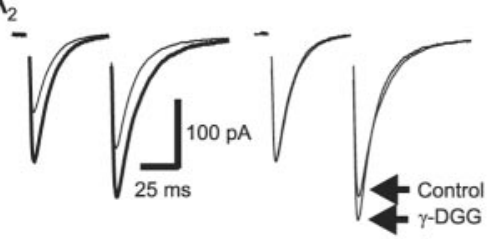

$\mathrm{B}_{1}$

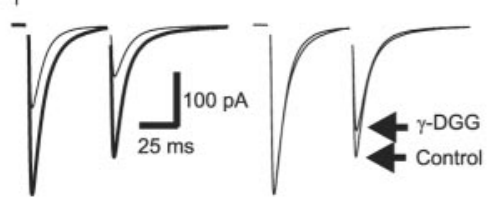

$\mathrm{B}_{2}$

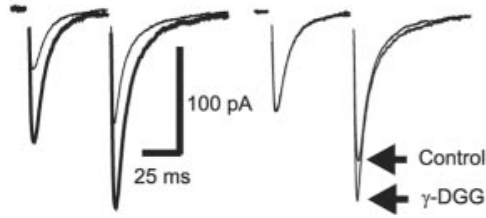

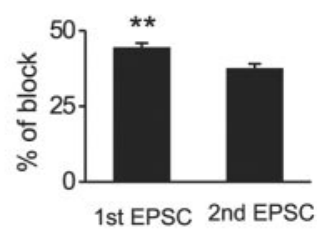
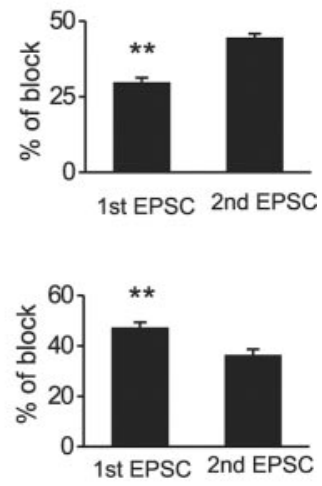

Figure 4. $\quad \gamma$-DGG differentially blocks EPSCs evoked during paired-pulse stimulation. $A 1, A 2$, Effects of $\gamma$-DGG on PPR at CI-AMPAR synapses displaying PPD (A1) or PPF (A2). Left traces, EPSCs evoked by two stimuli at an interval of $50 \mathrm{msec}$ in the absence (thick trace) and presence (thin trace) of $\gamma$-DGG (1 mM). Middle traces, EPSCs normalized to the peak of the first EPSC show significant differences in the degree of block of the second EPSC. Right, Summarized data, from synapses showing PPD $(n=5)$ and PPF $(n=5)$ reveal a differential block by $\gamma$-DGG of the first and second EPSC. B1, B2, Corresponding data from CP-AMPAR synapses showing PPD $(n=5)$ $(B 1)$ or PPF $(n=5)$ (B2). Data are arranged as described for Cl-AMPAR synapses in $A 1$ and $A 2$. Double asterisks indicate that the degree of block by $\gamma$-DGG of the first EPSC is significantly different from the second EPSC $(p<0.01)$.

the rise times of $\mathrm{EPSC}_{1}$ and $\mathrm{EPSC}_{2}$ and reduced the decay kinetics

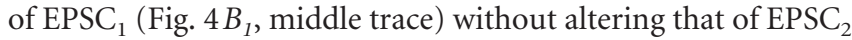
(Table 1). In contrast, at CP-AMPAR synapses showing PPF, $\gamma$-DGG inhibition of EPSC $_{1}(47.3 \pm 2.1 \%)$ was significantly larger than $\mathrm{EPSC}_{2}(36.4 \pm 2.3 \% ; n=5 ; p=0.003)$ (Fig. $\left.4 B_{2}\right)$. In addition, $\gamma$-DGG increased the rise times of $\mathrm{EPSC}_{1}$ and $\mathrm{EPSC}_{2}$ without changing their decay kinetics (Table 1). It is unlikely that $\gamma$-DGG directly altered glutamate release probability, because the CV of AMPA EPSCs was not altered by $\gamma$-DGG at either CIAMPAR (control, $0.31 \pm 0.04 ; \gamma$-DGG, $0.32 \pm 0.03 ; n=5 ; p=$ 0.81 ) or CP-AMPAR (control, $0.34 \pm 0.05 ; \gamma$-DGG, $0.37 \pm 0.06$; $n=5 ; p=0.93$ ) synapses. Together, the differential magnitude of $\gamma$-DGG block of EPSC Dersus $_{1}$ EPSC $_{2}$ is consistent with the hypothesis that differing glutamate transients are released by the first and second stimuli, possibly as a result of multivesicular release during transmission (Wadiche and Jahr, 2001), changes in fusion pore dynamics (Choi et al., 2000; Aravanis et al., 2003; Gandhi and Stevens, 2003), or glutamate pooling from multiple adjacent sites (Barbour and Hausser, 1997; Rusakov and Kullmann, 1998). It is important to point out that although these data are consistent with $\gamma$-DGG detecting changes in the synaptic glutamate transient, low-affinity antagonists can also reduce both postsynaptic receptor saturation and desensitization during paired-pulse protocols (Neher and Sakaba, 2001; Taschenberger

et al., 2002). Thus, caution must be exercised when interpreting such data.

\section{$\gamma$-DGG reveals a presynaptic locus of expression for CP-AMPAR LTD}

Having determined that the low-affinity antagonist $\gamma$-DGG reliably detects changes in glutamate transient concentrations at mossy fiber-interneuron synapses, we next determined whether LTD expression at CP-AMPAR and CI-AMPAR synapses resulted from a change in transmitter release. Specifically, if the released glutamate concentration in the synaptic cleft decreases after LTD expression, then the degree of $\gamma$-DGG inhibition would be expected to increase relative to controls. Therefore, comparison of the percentage change of $\gamma$-DGG inhibition of EPSCs before and after LTD induction may further elucidate the LTD expression loci at both CP-AMPAR and CI-AMPAR synapses.

To compare the magnitude of $\gamma$-DGG inhibition of mossy fiber-evoked AMPAR EPSCs before and after LTD expression, the experiment requires that we apply $\gamma$-DGG twice. As a control, we first confirmed that the sensitivity of $\gamma$-DGG-mediated inhibition was unchanged by repeated application. Application of $\gamma$-DGG $(1 \mathrm{~mm})$ reduced mossy fiber-evoked AMPA EPSCs by $52.9 \pm 1.7 \%(n=5)$. The effect of $\gamma$-DGG was fully reversible after 10-15 min of washout, and a second application of $\gamma$-DGG inhibited EPSCs to a similar extent $(53.9 \pm 1.6 \%$ reduction; $n=$ $5 ; p=0.87)$, indicating that $\gamma$-DGG sensitivity was unaltered after repeated applications.

Next, we measured the magnitude of $\gamma$-DGG inhibition before and after induction of LTD. At CI-AMPAR synapses (RI, $0.92 \pm 0.02 ; n=8)$, application of $\gamma$-DGG $(1 \mathrm{~mm})$ reduced EPSCs by $54.6 \pm 3.7 \%(n=8)$ in controls (Fig. $5 A)$. After washing out $\gamma$-DGG, HFS induced LTD; EPSC amplitude was reduced to $49.5 \pm 4.7 \%$ of controls $(n=8 ; p=0.001)$ measured $10 \mathrm{~min}$ after the end of the induction protocol (Fig. $5 A$, bottom). After LTD expression, a second application of $\gamma$-DGG inhibited EPSCs to $46.9 \pm 3.5 \%$ of controls $(n=8)$, a value similar to controls $(p=0.4)$ (Fig. $5 B, C)$. This result suggests that the glutamate concentration in the synaptic cleft was not changed after LTD expression at CI-AMPAR synapses.

At CP-AMPAR synapses (RI, $0.19 \pm 0.02 ; n=6), \gamma$-DGG inhibited EPSCs by $43.7 \pm 4.3 \%(n=6)$ in controls (Fig. $6 A)$. High-frequency stimulation induced LTD and reduced EPSC amplitude to $58.7 \pm 2.9 \%$ of controls $(n=6 ; p=0.002)$. After LTD expression, $\gamma$-DGG now inhibited the EPSC amplitude by $72.1 \pm 2.1 \%(n=6)$, a value significantly higher than that observed in controls $(p=0.0008$ ) (Fig. $6 B, C)$, suggesting that the glutamate concentration in the synaptic cleft was reduced after LTD expression.

Thus far, for ease of discussion, we focused on those synapses at the ends of the continuum that ranges from GluR2-lacking, CP-AMPAR synapses to GluR2-containing, CI-AMPAR synapses (Lei and McBain, 2002). For completeness, it is important to determine the LTD expression locus at that population of synapses comprising intermediate RIs [i.e., $0.7-0.3$ ( $\sim 25 \%$ of the total synapses studied)]. It is important to point out that we cannot determine whether these intermediate synapses reflect a homogeneous population of AMPA receptors with intermediate $\mathrm{Ca}^{2+}$ permeability or whether they represent synapses comprising both CI-AMPARs and CP-AMPARs in varying ratios, or multiple fibers activating different ratios of synapses composed of CP-AMPA or CI-AMPA receptors. In nine synapses (RIs between 0.3 and 0.7 ), the degree of change of $\gamma$-DGG block after LTD 
Table 1. EPSC parameters in $2.5 \mathrm{~mm} \mathrm{Ca}{ }^{2+}$

\begin{tabular}{|c|c|c|c|c|c|c|}
\hline & $\mathrm{EPSC}_{1}$ & & & $\mathrm{EPSC}_{2}$ & & \\
\hline & Control & $\gamma$-DGG $(1 \mathrm{~mm})$ & $\mathrm{p}$ & Control & $\gamma$-DGG $(1 \mathrm{~mm})$ & $p$ \\
\hline CI-AMPAR & & & & & & \\
\hline PPD & & & & & & \\
\hline Rise time (msec) & $0.56 \pm 0.07$ & $0.63 \pm 0.08$ & 0.019 & $0.76 \pm 0.06$ & $0.79 \pm 0.07$ & 0.048 \\
\hline Decay time (msec) ${ }^{a}$ & $17.5 \pm 1.6$ & $14.4 \pm 1.8$ & 0.002 & $21.8 \pm 3.5$ & $13.6 \pm 1.2$ & 0.06 \\
\hline PPF & & & & & & \\
\hline Rise time (msec) & $0.61 \pm 0.05$ & $0.67 \pm 0.05$ & 0.019 & $0.72 \pm 0.05$ & $0.77 \pm 0.06$ & 0.037 \\
\hline Decay time (msec) & $12.3 \pm 1.5$ & $12.0 \pm 1.6$ & 0.6 & $9.8 \pm 2.1$ & $13.0 \pm 1.2$ & 0.22 \\
\hline CP-AMPAR & & & & & & \\
\hline PPD & & & & & & \\
\hline Rise time (msec) & $0.54 \pm 0.05$ & $0.60 \pm 0.05$ & 0.02 & $0.66 \pm 0.04$ & $0.72 \pm 0.05$ & 0.02 \\
\hline Decay time (msec) & $7.1 \pm 0.6$ & $5.8 \pm 0.3$ & 0.02 & $9.8 \pm 1.1$ & $8.4 \pm 1.3$ & 0.32 \\
\hline PPF & & & & & & \\
\hline Rise time (msec) & $0.53 \pm 0.04$ & $0.73 \pm 0.03$ & 0.001 & $0.62 \pm 0.04$ & $0.79 \pm 0.04$ & 0.003 \\
\hline Decay time (msec) & $8.2 \pm 1.5$ & $8.3 \pm 1.3$ & 0.75 & $16.2 \pm 0.40$ & $15.1 \pm 1.9$ & 0.61 \\
\hline
\end{tabular}

${ }^{a}$ The decay time constant represents the weighted mean. $n=5$ for all conditions. $p$ values were determined by paired $t$ test.

A
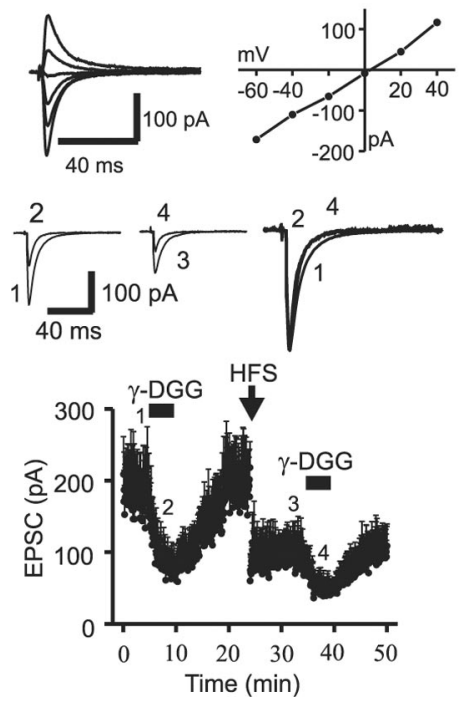

B

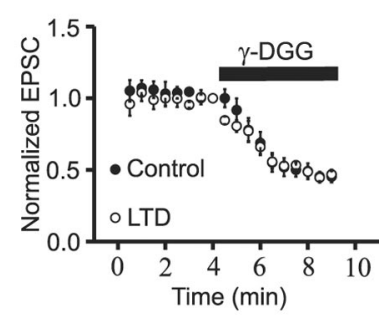

C

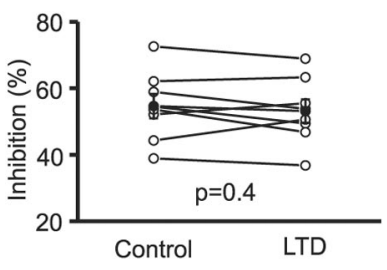

A
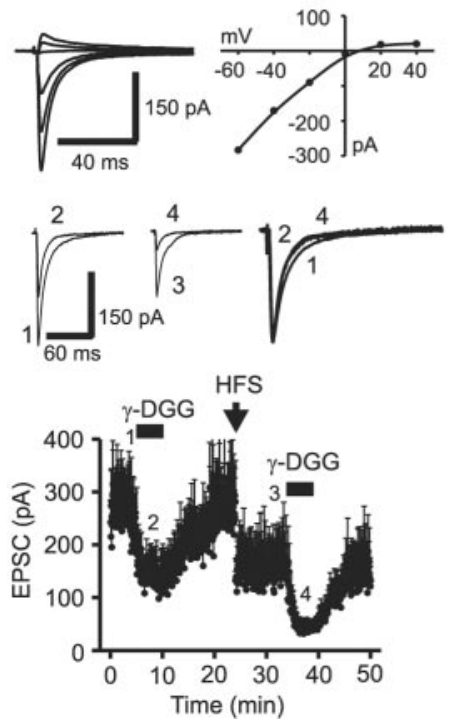

B

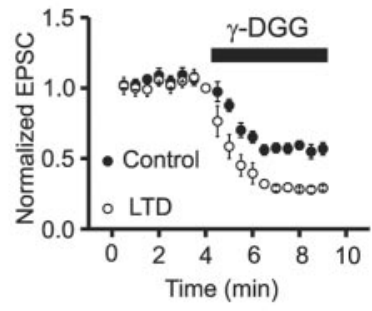

C

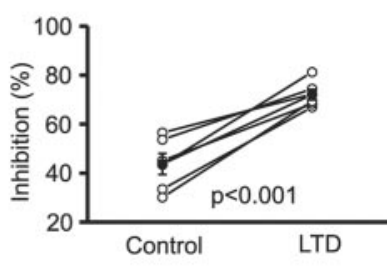

Figure 5. LTD at Cl-AMPAR synapses does not involve a change in transmitter release. $A$, Top, EPSCs recorded at holding potentials between -60 and $+40 \mathrm{mV}$ (left) and their corresponding $I-V$ relationship (right). A linear $I-V$ curve $(R I, 1.19)$ suggests that this synapse comprises CI-AMPARs. In all experiments, dl-APV was initially included to block NMDARs for the construction of the AMPAR-EPSC I-V relationship. After determination of the RI, APV was then washed out. Middle, Averages of 10 consecutive EPSCs taken at time points indicated in the bottom dot plot. The right panel shows the normalization of control averaged EPSCs (1) and averaged EPSCS recorded during the first (2) and second (4) application of $\gamma$-DGG. Bottom, Mean time courses of EPSCs averaged from eight neurons. In the control epoch, $\gamma$-DGG $(1 \mathrm{~mm})$ was applied to measure the basal level of inhibition. After complete washout, the high-frequency LTD induction protocol (HFS, $100 \mathrm{~Hz}$ for 1 sec, repeated 3 times at an interval of 10 sec) was applied. After LTD had stabilized (3), the same concentration of $\gamma$-DGG was applied for a second time. B, EPSCs obtained during the two applications of $\gamma$-DGG were normalized to the controls and overlain to illustrate the degree of $\gamma$-DGG inhibition before and after induction of LTD. A comparable degree of $\gamma$-DGG inhibition was observed before and after induction of LTD. C, $\gamma$-DGG-mediated inhibition from eight individual CI-AMPAR synapses before and after the induction of LTD. The filled circles are averages of $\gamma$-DGG inhibition before and after the induction of LTD. Open circles are data obtained from individual experiments. Note that the percentage of inhibition by $\gamma$-DGG was not significantly different before and after the induction of LTD.

expression was intermediate $(49.7 \pm 1.7 \%$ for control $\gamma$-DGG block vs $63.1 \pm 3.1 \%$ after LTD; $p=0.007)$ to the values obtained for synapses at the two extremes. These data suggest that a presynaptic reduction in transmitter release also contributes to expression of LTD at those synapses with weakly rectifying $I-V$

Figure 6. LTD at CP-AMPAR synapses involves a reduction in transmitter release. $A$, Top, EPSCs recorded at holding potentials between -60 and $+40 \mathrm{mV}$ (left) and the corresponding $I-V$ relationship (right). An inwardly rectifying $I-V$ relationship suggests that this synapse comprised CP-AMPARs. dl-APV was included for the entire period of the experiment to isolate CP-AMPAR-dependent LTD. Middle, Averaged current traces from 10 EPSCs taken at the time points indicated in the dot plot (bottom). The right traces show the normalization of control EPSCS (1) and the EPSCs recorded in the presence of $\gamma$-DGG (2 and 4). Bottom, Time course of inhibition by $\gamma$-DGG (1 mM) ( 2 and 4 ) before (1) and (3) after induction of LTD from six neurons. $B$, EPSCS recorded during the two applications of $\gamma$-DGG were normalized to their respective controls for ease of comparison of the degree of $\gamma$-DGG block before and after the induction of LTD. C, $\gamma$-DGG-mediated inhibition from six individual CP-AMPAR synapses before and after the induction of LTD. The filled circles are averages of $\gamma$-DGG inhibition before and after the induction of LTD. Open circles are data obtained from individual experiments. Note that the percentage of inhibition by $\gamma$-DGG was significantly increased after the induction of LTD at CP-AMPAR synapses.

relationships; however, a contribution of postsynaptic mechanisms cannot be fully excluded. These data raise an important issue that suggests that mixed (presynaptic and postsynaptic) forms of LTD cannot be excluded at individual synapses, and although the locus of expression is mainly presynaptic at $\mathrm{CP}$ AMPAR synapses and mainly postsynaptic at CI-AMPAR synapses, overlapping contributions from both forms of LTD cannot be ruled out. 
NSF-AP2 inhibitory peptide confirms a postsynaptic locus of expression for NMDAR-dependent LTD at

\section{CI-AMPAR synapses}

Postsynaptic AMPAR translocation is an important mechanism in NMDAR-dependent LTD expression at synapses onto pyramidal neurons (Carroll et al., 1999; Lüscher et al., 1999; Lüthi et al., 1999; Man et al., 2000; Matsuda et al., 2000; Wang and Linden, 2000; Sheng and Kim, 2002; Song and Huganir, 2002). At Schaffer collateral-CA1 pyramidal neuron synapses, NMDARdependent LTD expression involves a pool of AMPARs regulated by both NSF-GluR2 (Lüscher et al., 1999; Lüthi et al., 1999; Noel et al., 1999) and clathrin adaptor AP2-GluR2 (Lee et al., 2002) interactions. NMDAR-dependent LTD-induced endocytosis of AMPARs is dependent on both $\mathrm{Ca}^{2+}$ and the activity of protein phosphatase 1 or 2A (Beattie et al., 2000; Ehlers, 2000). We next tested whether expression of LTD at either CI-AMPAR or CPAMPAR synapses similarly involved a translocation of AMPARs using the broad-spectrum NSF-AP2 inhibitory peptide, commonly referred to as pep2m (Lüscher et al., 1999; Lüthi et al., 1999; Shi et al., 2001; Lee et al., 2002).

Infusion of pep $2 \mathrm{~m}$ into the cells via the recording pipette did not significantly influence evoked AMPA EPSC amplitudes (note that it only affected CI-AMPAR EPSCs; see below for details) until $>8$ min after formation of the whole-cell recording. Taking advantage of this initial control period, we first constructed $I-V$ curves and calculated the RI of evoked EPSCs to determine the $\mathrm{Ca}^{2+}$-permeable nature of AMPARs. At CI-AMPAR synapses (RI, $0.96 \pm 0.05 ; n=7$ ) (Fig. $7 A$, top), infusion of pep $2 \mathrm{~m}(0.5$ $\mathrm{mM}$ ) not only decreased EPSC amplitude to $67.7 \pm 4.5 \%$ of controls (measured $38 \mathrm{~min}$ after the formation of whole-cell recording; $n=7 ; p=0.0004$ ) (Fig. $7 B$ ) but occluded LTD expression (Fig. $7 A, B$ ). EPSC amplitude was $67.7 \pm 4.5 \%$ of controls before LTD induction versus $64.5 \pm 6.8 \%$ of controls at $20 \mathrm{~min}$ after LTD expression $(n=7 ; p=0.35)$ (Fig. $7 B)$. Together with the results from the $\gamma$-DGG inhibition experiment, these data strongly suggest that the expression of NMDAR-dependent LTD at CI-AMPAR synapses is postsynaptic and involves a pool of AMPARs regulated by the NSF-AP2-GluR2 interaction.

In contrast, pep $2 \mathrm{~m}$ did not appreciably inhibit EPSC amplitude at CP-AMPAR synapses even $\sim 40$ min after formation of the whole-cell recording $(102.2 \pm 9.9 \%$ of the initial EPSC amplitude; $n=5 ; p=0.84$ ) (Fig. 7C,D), suggesting that there was no NSF-AP2-GluR2 interaction at CP-AMPAR synapses. These data are consistent with results obtained from CA1 hippocampal pyramidal neurons containing a deletion of the GluR2 subunit (Shi et al., 2001) and confirm that the effect of the NSF-AP2 inhibitory peptide is specific for interactions mediated by GluR2 at GluR2-containing receptors (Sheng and Kim, 2002; Song and Huganir, 2002). Furthermore, application of the LTD induction protocol still depressed transmission at CP-AMPAR synapses (RI, $0.19 \pm 0.03 ; n=5$ ) (Fig. 7C,D). EPSC amplitude was reduced by $49.5 \pm 7.7 \%(n=5 ; p=0.003) 20 \mathrm{~min}$ after LTD expression. Together with the result from the $\gamma$-DGG inhibition experiment, these results indicate that the LTD expression at CPAMPAR synapses is consistent with a reduction in transmitter release with no involvement of postsynaptic AMPA receptor translocation.

Finally, in an attempt to separate the NSF-GluR2 interaction (i.e., the EPSC amplitude "rundown") from the clathrin adaptor AP2-dependent translocation of AMPARs (i.e., one potential mechanism for LTD expression), we used an AP2-specific blocking peptide (pep- $\Delta$ A849-Q853) (Lee et al., 2002). At Schaffer collateral-CA1 pyramidal neuron synapses, this peptide does not
Cl-AMPAR synapse

A
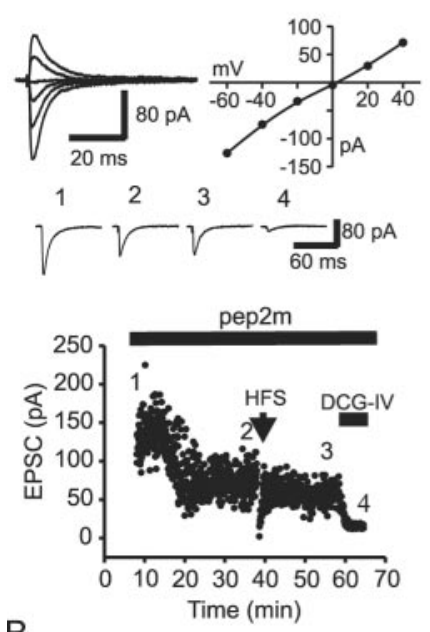

B

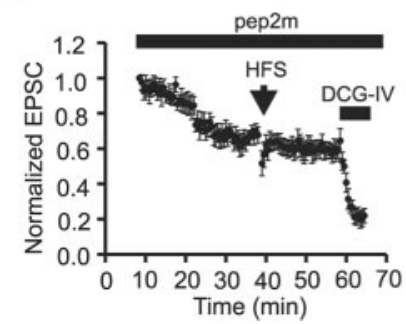

CP-AMPAR synapse

C
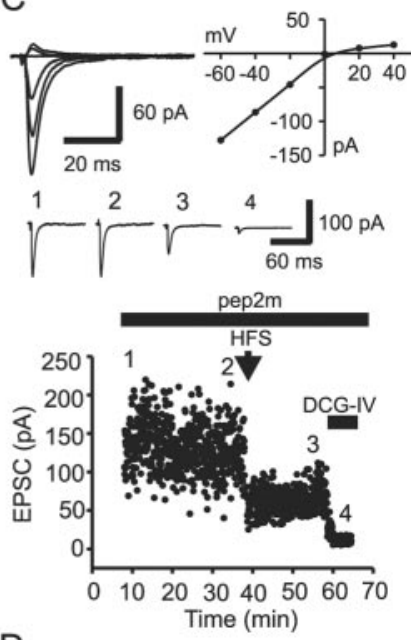

D

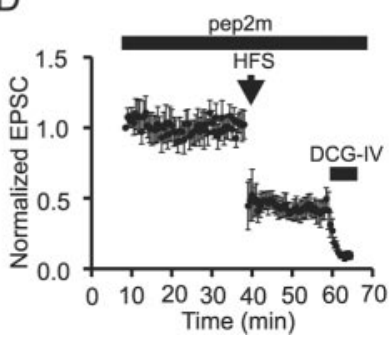

Figure 7. Postsynaptic AMPAR trafficking contributes to expression of LTD at CI-AMPAR synapses. The NSF-AP2-inhibitory peptide pep $2 \mathrm{~m}(0.5 \mathrm{~mm})$ was included in the recording pipettes for all experiments. A, Cl-AMPAR synapse. Top, EPSCs evoked at holding potentials between -60 and $+40 \mathrm{mV}$, recorded during the first $8 \mathrm{~min}$ after formation of whole-cell configuration (left) and the corresponding $I-V$ relationship (right) from a Cl-AMPAR synapse. dl-APV was included for the period of RI determination. Middle, Averaged current traces from 10 EPSCs taken at the time points indicated in the dot plot below. Bottom, Dot plot of EPSC amplitude indicating the time course of the experiment. The first $8 \mathrm{~min}$ after the formation of wholecell recording were used to construct the $I-V$ relationship and identify the $\mathrm{Ca}^{2+}$-permeable nature of the AMPAR-mediated EPSC. Inclusion of the NSF inhibitory peptide inhibited EPSCs at this Cl-AMPAR synapse and occluded expression of LTD. DCG-IV was included in the extracellular solution at the end of the recording to confirm that EPSCs were mossy fiber in nature. B, Averaged data from seven CI-AMPAR synapses illustrate the reduction of EPSC amplitude and the concomitant occlusion of LTD. C, CP-AMPAR synapse. The I-V relationship and the time course of evoked EPSCs at a CP-AMPAR synapse are shown. Organization of data is identical to that shown in $A$ and $B$. Inclusion of the NSF inhibitory peptide was without effect on both the EPSC amplitude and the ability to express LTD at CP-AMPAR synapses. D, Averaged data from five CP-AMPAR synapses.

affect basal synaptic transmission but completely abolishes lowfrequency stimulation-induced LTD. In contrast, at mossy fiberinterneuron synapses, although pep- $\Delta$ A849-Q853 did not impact basal synaptic transmission (CI-AMPAR synapse, $97.6 \pm$ $2.1 \%, n=5, p=0.32$; CP-AMPAR synapse, $92.0 \pm 6.0 \%, p=$ $0.26, n=5)$, it also failed to block LTD expression at both synapse types (CI-AMPAR synapse, $52.3 \pm 6.8 \%, n=5, p=0.002$; CPAMPAR synapse, $49.5 \pm 6.1 \%, p=0.001, n=5$ ), suggesting that the AP2-dependent pathway may not be necessary for LTD expression at interneuron synapses, consistent with the lack of detectable protein phosphatase $2 \mathrm{~B}$ at these synapses (Sik et al., 1998).

\section{Discussion}

We used three approaches to explore the expression locus of two forms of mossy fiber-inhibitory interneuron LTD: (1) conven- 
tional analysis of synaptic parameters, including CV analysis, PPR, and analysis of both AMPA and NMDA receptor-mediated EPSCs; (2) application of a low-affinity glutamate receptor antagonist to probe changes in the synaptic glutamate transient concentration; and (3) infusion of NSF-GluR2- or AP2-GluR2interfering peptides into cells to explore potential postsynaptic mechanisms. All three approaches point to the same conclusion: that the expression locus of each form of mossy fiber-interneuron LTD occurs on opposite sides of the synapse.

LTD expression at CP-AMPAR synapses was associated with increases in both the CV and PPR. Furthermore, the concentration of cleft glutamate, assessed by measuring changes in $\gamma$-DGG inhibition, was reduced after LTD expression. Finally, postsynaptic introduction of pep $2 \mathrm{~m}$, an NSF-AP2 inhibitory peptide, failed to influence the expression of LTD at CP-AMPAR synapses, suggesting that postsynaptic AMPAR translocation is unlikely to be involved. Together, these results indicate that LTD expression at CP-AMPAR synapses is presynaptic in origin and is reminiscent of mossy fiber-basket cell LTD (Alle et al., 2001). In contrast, LTD expression at CI-AMPAR synapses did not alter the CV or paired-pulse ratio, and the magnitude of $\gamma$-DGG inhibition was also unaltered after LTD expression, arguing against presynaptic expression. Postsynaptic expression was further supported by experiments involving postsynaptic infusion of pep $2 \mathrm{~m}$, which occluded LTD expression. Together, these results suggest that LTD expression at CI-AMPAR synapses is postsynaptic and involves a pool of AMPARs regulated by NSF-AP2-GluR2 interaction.

What is the mechanism whereby LTD results in decreased glutamate release at mossy fiber-CP-AMPAR synapses? If transmission at CP-AMPAR synapses occurred via univesicular release [i.e., a "one release site-one vesicle" mechanism (Redman 1990; Korn et al., 1994)] and LTD expression involved a reduction in transmitter release probability, then a change in the magnitude of $\gamma$-DGG block would not be expected to occur after LTD expression. Clearly, such a mechanism is unlikely to account for the increased block by $\gamma$-DGG after CP-AMPAR LTD expression. We suggest three possible scenarios to explain the present observations: (1) Under control conditions, transmission at CPAMPAR synapses may result from multivesicular release [i.e., more than one vesicle can be released from a single synapse after a stimulus (Tong and Jahr, 1994; Auger et al., 1998; Prange and Murphy, 1999)]. LTD expression may therefore arise from a reduced likelihood of multiple exocytotic events at a single release site as the probability of release is reduced after LTD induction (Wadiche and Jahr, 2001), resulting in a lowered glutamate transient. Evidence for multivesicular release (Jahr, 2003) has been provided at an increasing number of disparate synapses, including the neuromuscular junction (Heuser et al., 1979), cerebellar stellate, and basket cell inhibitory synapses (Auger et al., 1998), excitatory synapses onto hippocampal neurons (Tong and Jahr, 1994; Oertner et al., 2002; Hallermann et al., 2003), and climbing fiber-Purkinje cell synapses (Wadiche and Jahr, 2001). Recent evidence suggests that the release rate at the large mossy fiberCA3 pyramidal neuron terminal is also consistent with multivesicular release (Hallermann et al., 2003). The differential degree of block by $\gamma$-DGG during paired-pulse protocols also suggests that multivesicular release may occur at the smaller mossy fiberinterneuron synapses under basal conditions. (2) Alternatively, CP-AMPAR LTD may arise from changes in fusion pore dynamics or reversal of the "kiss and run" hypothesis (Choi et al., 2000; Aravanis et al., 2003; Gandhi and Stevens, 2003), where LTD results from an incomplete emptying of vesicular glutamate. In- creasing evidence suggests that multiple exocytotic states exist within single synapses and that mechanisms that shift the probability of each state will shape transmitter release profile at individual synapses. (3) Under normal conditions, mossy fiber transmission at CP-AMPAR synapses may result from glutamate pooling from multiple adjacent sites. A reduction in release probability will reduce the likelihood that adjacent synapses release transmitter simultaneously, consequently reducing the transmitter pool (Barbour and Hausser, 1997; Rusakov and Kullmann, 1998). Support for this hypothesis comes from consideration of the mossy fiber-filopodial architecture (Acsady et al., 1998) and their known quantal properties (Lawrence et al., 2004). Filopodia primarily synapse onto smooth, spine-free dendritic segments or arrange in clusters onto the characteristic long and thin dendritic spines of inhibitory interneurons. On occasion, multiple filopodia emanating from a single large mossy bouton make contact with a single postsynaptic interneuron. Qualitatively, the lowaffinity antagonist technique by itself cannot distinguish between these three interpretations, and future experiments will attempt to elucidate the precise mechanism of LTD expression at CPAMPAR synapses.

Postsynaptic $\mathrm{Ca}^{2+}$ elevation, presumably through NMDA and $\mathrm{Ca}^{2+}$-permeable AMPA receptors, is required for LTD induction at CI-AMPAR and CP-AMPAR synapses, respectively (Lei and McBain, 2002). Our results suggest that $\mathrm{Ca}^{2+}$ influx through NMDARs leads to the translocation of AMPARs and LTD expression only at CI-AMPAR synapses. This mechanism is reminiscent of the NMDAR-dependent LTD observed at Schaffer collateral-CA1 pyramidal neuron synapses, which involves postsynaptic AMPAR trafficking (Lüscher et al., 1999; Lüthi et al., 1999; Beattie et al., 2000; Lee et al., 2002; Sheng and Kim, 2002; Song and Huganir, 2002). However, it is unclear how $\mathrm{Ca}^{2+}$ entry via NMDARs leads to the translocation of AMPARs at interneuron-CI-AMPAR synapses. In cultured hippocampal neurons, activation of NMDARs triggers AMPAR endocytosis through $\mathrm{Ca}^{2+}$ influx and activation of the $\mathrm{Ca}^{2+}$-dependent protein phosphatase calcineurin (Beattie et al., 2000). However, calcineurin is absent from hippocampal interneurons (Sik et al., 1998), making it an unlikely target for $\mathrm{Ca}^{2+}$ influx at CI-AMPAR synapses. Consistent with this hypothesis was the failure of the AP2-GluR2-interfering peptide (Lee et al., 2002) to block LTD induction. This suggests that although NMDAR-dependent LTD shares many of the features of LTD observed at principal cell synapses, an identical mechanism would appear unlikely. Given that diverse intracellular signals are involved in AMPAR endocytosis (Beattie et al., 2000; Chung et al., 2000; Ehlers, 2000; Lin et al., 2000; Matsuda et al., 2000), it would not be surprising to see alternative intracellular signals involved in synaptic trafficking of AMPARs at CI-AMPAR synapses.

Postsynaptic NMDAR-dependent LTD of excitatory transmission onto CA1 pyramidal neurons is associated with an increase in failure rate (Stevens and Wang, 1994) and a change in CV (Selig et al., 1995), consistent with the silencing of "active" synapses by receptor internalization (Malenka and Siegelbaum, 2001; Sheng and Kim, 2002). In contrast, the NMDARdependent LTD observed at interneuron synapses was not associated with changes in the CV. How can we explain these two disparate observations? The presence of "silent" synapses on principal cells has been supported by considerable anatomical and physiological evidence, whereas little evidence exists for anatomically silent synapses onto interneurons. Furthermore, excitatory synapses onto interneurons have an AMPAR distribution with a relatively small variability and contain on average four 
times as many receptors as excitatory synapses onto CA3 pyramidal cell spines (Nusser et al., 1998). Such an anatomical arrangement suggests that postsynaptic LTD at interneuron synapses may reduce AMPAR content but fail to "silence" active synapses. In this scenario, LTD would result as a reduction in EPSC amplitude without a change in CV.

Interestingly, $\mathrm{Ca}^{2+}$ influx via CP-AMPARs does not engage translocation of AMPA receptors, and LTD expression appears to be entirely presynaptic. What then is the link between postsynaptic induction and presynaptic expression? Laezza et al. (1999) speculated that entry of $\mathrm{Ca}^{2+}$ through CP-AMPARs at collateral synapses onto stratum radiatum interneurons may trigger a synaptic shape change that enables access of released glutamate to presynaptic mGluRs, or cause release of a retrograde messenger that cooperates with presynaptic mGluR activation to suppress transmitter release, perhaps via the recently described transsynaptic EphB-Ephrin receptor signaling system (Contractor et al., 2002). Future experiments will elucidate these mechanisms.

Finally, these experiments raise an intriguing hypothesis that mossy fiber innervation of CA3 stratum lucidum interneurons occurs via two parallel systems: one linked to CP-AMPAR synapses that contain low levels of NR2B-containing NMDARs and a second mossy fiber system that engages CI-AMPAR synapses, which contain a significant NMDAR contribution. Highfrequency stimulation of CP-AMPAR synapses results in a presynaptic form of LTD expression associated with a reduction in both synaptic glutamate transient and release probability, which alters short-term plasticity at these synapses. However, LTD induction and expression at CI-AMPAR synapses appears to be entirely postsynaptic and to involve translocation of AMPAR subunits. Of particular interest at synapses with an intermediate RI profile (i.e., RIs of 0.3-07), the presynaptic form of LTD expression predominates, suggesting that these synapses may "default" to a plasticity involving an alteration in transmitter release probability. How these properties of distinct short-term and long-term plasticity map onto the mossy fiber-CA3 network dialogue is unclear, but mechanisms that alter the mean EPSC amplitude without changing the variance (postsynaptic LTD) will have a profoundly different impact from depression associated with a concomitant change in variance (presynaptic LTD) (Aradi et al., 2002).

\section{References}

Acsady L, Kamondi A, Sik A, Freund T, Buzsaki G (1998) GABAergic cells are the major postsynaptic targets of mossy fibers in the rat hippocampus. J Neurosci 18:3386-3403.

Alle H, Jonas P, Geiger JRP (2001) PTP and LTP at a hippocampal mossy fiber-interneuron synapse. Proc Natl Acad Sci USA 98:14708-14713.

Aradi I, Santhakumar V, Chen K, Soltesz I (2002) Postsynaptic effects of GABAergic synaptic diversity: regulation of neuronal excitability by changes in IPSC variance. Neuropharmacology 43:511-522.

Aravanis AM, Pyle JL, Tsien RW (2003) Single synaptic vesicles fusing transiently and successively without loss of identity. Nature 423:643-647.

Auger C, Kondo S, Marty A (1998) Multivesicular release at single functional synaptic sites in cerebellar stellate and basket cells. J Neurosci 18:4532-4547.

Barbour B, Hausser M (1997) Intersynaptic diffusion of neurotransmitter. Trends Neurosci 20:377-384.

Beattie EC, Carroll RC, Yu X, Morishita W, Yasuda H, von Zastrow M, Malenka RC (2000) Regulation of AMPA receptor endocytosis by a signaling mechanism shared with LTD. Nat Neurosci 3:1291-1300.

Bischofberger J, Jonas P (2002) TwoB or not TwoB: differential transmission at glutamatergic mossy fiber-interneuron synapses in the hippocampus. Trends Neurosci 25:600-603.

Carroll RC, Lissin DV, von Zastrow M, Nicoll RA, Malenka RC (1999)
Rapid redistribution of glutamate receptors contributes to long-term depression in hippocampal cultures. Nat Neurosci 2:454-460.

Choi S, Klingauf J, Tsien RW (2000) Postfusional regulation of cleft glutamate concentration during LTP at "silent synapses." Nat Neurosci 3:330-336.

Chung HJ, Xia J, Scannevin RH, Zhang X, Huganir RL (2000) Phosphorylation of the AMPA receptor subunit GluR2 differentially regulates its interaction with PDZ domain-containing proteins. J Neurosci 20:7258-7267. 100:4885-4890.

Contractor A, Rogers C, Maron C, Henkemeyer M, Swanson GT, Heinemann SF (2002) Trans-synaptic Eph receptor-ephrin signaling in hippocampal mossy fiber LTP. Science 296:1864-1869.

Ehlers MD (2000) Reinsertion or degradation of AMPA receptors determined by activity-dependent endocytic sorting. Neuron 28:511-525.

Gandhi SP, Stevens CF (2003) Three modes of synaptic vesicular recycling revealed by single-vesicle imaging. Nature 423:607-613.

Hallermann S, Pawlu C, Jonas P, Heckmann M (2003) A large pool of releasable vesicles in a cortical glutamatergic synapse. Proc Natl Acad Sci USA 100:8975-8980.

Harris EW, Cotman CW (1986) Long-term potentiation of guinea pig mossy fiber responses is not blocked by $N$-methyl-D-aspartate antagonists. Neurosci Lett 70:132-137.

Heuser JE, Reese TS, Dennis MJ, Jan Y, Jan L, Evans L (1979) Synaptic vesicle exocytosis captured by quick freezing and correlated with quantal transmitter release. J Cell Biol 81:275-300.

Jahr CE (2003) Drooling and stuttering, or do synapses whisper? Trends Neurosci 26:7-9.

Kamiya H, Shinozaki H, Yamamoto C (1996) Activation of metabotropic glutamate receptor type $2 / 3$ suppresses transmission at rat hippocampal mossy fibre synapses. J Physiol (Lond) 493:447-455.

Kim J, Alger BE (2001) Random response fluctuations lead to spurious paired-pulse facilitation. J Neurosci 21:9608-9618.

Korn H, Sur C, Charpier S, Legendre P, Faber DS (1994) The one-vesicle hypothesis and multivesicular release. Adv Second Messenger Phosphoprotein Res 29:301-322.

Laezza F, Doherty JJ, Dingledine R (1999) Long-term depression in hippocampal interneurons: joint requirement for pre- and postsynaptic events. Science 285:1411-1414.

Lawrence JJ, McBain CJ (2003) Containing the detonation-feedforward inhibition in the CA3 hippocampus. Trends Neurosci 26:631-640.

Lawrence JJ, Grinspan Z, McBain CJ (2004) Quantal transmission at mossy fibre targets in the CA3 region of the rat hippocampus. J Physiol (Lond) 554:175-193.

Lee SH, Liu L, Wang YT, Sheng M (2002) Clathrin adaptor AP2 and NSF interact with overlapping sites of GluR2 and play distinct roles in AMPA receptor trafficking and hippocampal LTD. Neuron 36:661-674.

Lei S, McBain CJ (2002) Distinct NMDA receptors provide differential modes of transmission at mossy fiber-interneuron synapses. Neuron 33:921-933.

Lei S, McBain CJ (2003) GABA B receptor modulation of excitatory and inhibitory synaptic transmission onto rat CA3 hippocampal interneurons. J Physiol (Lond) 546:439-453.

Lin JW, Ju W, Foster K, Lee SH, Ahmadian G, Wyszynski M, Wang YT, Sheng M (2000) Distinct molecular mechanisms and divergent endocytotic pathways of AMPA receptor internalization. Nat Neurosci 3:1282-1290.

Liu SJ, Cull-Candy SG (2000) Synaptic activity at calcium-permeable AMPA receptors induces a switch in receptor subtype. Nature 25:454-458.

Liu SJ, Cull-Candy SG (2002) Activity-dependent change in AMPA receptor properties in cerebellar stellate cells. J Neurosci 22:3881-3889.

Liu G, Choi S, Tsien RW (1999) Variability of neurotransmitter concentration and nonsaturation of postsynaptic AMPA receptors at synapses in hippocampal cultures and slices. Neuron 22:395-409.

Lüscher C, Xia H, Beattie EC, Carroll RC, von Zastrow M, Malenka RC, Nicoll RA (1999) Role of AMPA receptor cycling in synaptic transmission and plasticity. Neuron 24:649-658.

Lüthi A, Chittajallu R, Duprat F, Palmer MJ, Benke TA, Kidd FL, Henley JM, Isaac JT, Collingridge GL (1999) Hippocampal LTD expression involves a pool of AMPARs regulated by the NSF-GluR2 interaction. Neuron 24:389-399.

Maccaferri G, Toth K, McBain CJ (1998) Target-specific expression of presynaptic mossy fiber plasticity. Science 279:1368-1370. 
Malenka RC, Siegelbaum SA (2001) Synaptic plasticity: diverse targets and mechanisms for regulating synaptic efficacy. In: Synapses (Cowan WC, Sudhof TC, Stevens CF, eds), pp 393-453. Baltimore: Johns Hopkins.

Man HY, Lin JW, Ju WH, Ahmadian G, Liu L, Becker LE, Sheng M, Wang YT (2000) Regulation of AMPA receptor-mediated synaptic transmission by clathrin-dependent receptor internalization. Neuron 25:649-662.

Matsuda S, Launey T, Mikawa S, Hirai H (2000) Disruption of AMPA receptor GluR2 clusters following long-term depression induction in cerebellar Purkinje neurons. EMBO J 19:2765-2774.

Neher E, Sakaba T (2001) Combining deconvolution and noise analysis for the estimation of transmitter release rates at the calyx of Held. J Neurosci 21:444-461.

Nicoll RA, Malenka RC (1995) Contrasting properties of two forms of longterm potentiation in the hippocampus. Nature 377:115-118.

Noel J, Ralph GS, Pickard L, Williams J, Molnar E, Uney JB, Collingridge GL, Henley JM (1999) Surface expression of AMPA receptors in hippocampal neurons is regulated by an NSF-dependent mechanism. Neuron 23:365-376.

Nusser Z, Lujan R, Laube G, Roberts JDB, Molnar E, Somogyi P (1998) Cell type and pathway dependence of synaptic AMPA receptor number and variability in the hippocampus. Neuron 21:545-559.

Oertner TG, Sabatini BL, Nimchinsky EA, Svoboda K (2002) Facilitation at single synapses probed with optical quantal analysis. Nat Neurosci 5:657-664.

Prange O, Murphy TH (1999) Analysis of multiquantal transmitter release from single cultured cortical neuron terminals. J Neurophysiol 81:1810-1817.

Redman S (1990) Quantal analysis of synaptic potentials in neurons of the central nervous system. Physiol Rev 70:165-198.

Rusakov DA, Kullmann DM (1998) Extrasynaptic glutamate diffusion in the hippocampus: ultrastructural constraints, uptake, and receptor activation. J Neurosci 18:3158-3170.

Selig DK, Hjelmstad GO, Herron C, Nicol RA, Malenka RC (1995) Independent mechanisms for long-term depression of AMPA and NMDA responses. Neuron 15:417-426.
Shen Y, Hansel C, Linden DJ (2002) Glutamate release during LTD at cerebellar climbing fiber-Purkinje cell synapses. Nat Neurosci 5:725-726.

Sheng M, Kim MY (2002) Postsynaptic signaling and plasticity mechanisms. Science 298:776-780.

Shi S, Hayashi Y, Esteban JA, Malinow R (2001) Subunit-specific rules governing AMPA receptor trafficking to synapses in hippocampal pyramidal neurons. Cell 105:331-343.

Sik A, Hajos N, Gulacsi A, Mody I, Freund TF (1998) The absence of a major $\mathrm{Ca}^{2+}$ signaling pathway in GABAergic neurons of the hippocampus. Proc Natl Acad Sci USA 95:3245-3250.

Song I, Huganir RL (2002) Regulation of AMPA receptors during synaptic plasticity. Trends Neurosci 25:578-588.

Stevens CF, Wang Y (1994) Changes in reliability of synaptic function as a mechanism for plasticity. Nature 79:365-375.

Taschenberger H, Leao RM, Rowland KC, Spirou GA, von Gersdorff H (2002) Optimizing synaptic architecture and efficiency for highfrequency transmission. J Neurosci 36:1127-1143.

Tong G, Jahr CE (1994) Multivesicular release from excitatory synapses of cultured hippocampal neurons. Neuron 12:51-59.

Toth K, McBain CJ (1998) Afferent-specific innervation of two distinct AMPA receptor subtypes on single hippocampal interneurons. Nat Neurosci 1:572-578.

Toth K, Suares G, Lawrence JJ, Philips-Tansey E, McBain CJ (2000) Differential mechanisms of transmission at three types of mossy fiber synapse. J Neurosci 20:8279-8289.

Wadiche JI, Jahr CE (2001) Multivesicular release at climbing fiberPurkinje cell synapses. Neuron 32:301-313.

Wang YT, Linden DJ (2000) Expression of cerebellar long-term depression requires postsynaptic clathrin-mediated endocytosis. Neuron 25:635-647.

Yeckel MF, Kapur A, Johnston D (1999) Multiple forms of LTP in hippocampal CA3 neurons use a common postsynaptic mechanism. Nat Neurosci 2:625-633.

Zalutsky RA, Nicoll RA (1990) Comparison of two forms of long-term potentiation in single hippocampal neurons. Science 248:1619-1624. 